\title{
Lichen planus induced by radiotherapy
}

\author{
M. Pretel and A. España
}

Department of Dermatology, University Clinic of Navarra, Pamplona, Navarra, Spain

E-mail: maiderpretel@yahoo.es

Lichen planus (LP) is an inflammatory dermatosis of autoimmune origin, which affects the skin and mucosae. Although the aetiology of LP remains uncertain, certain viruses (eg, hepatitis B and C), contact with diverse substances (eg, mercury and copper) and neoplasias have been linked to the condition.

A 44-year-old woman presented at our department in 2003 with multiple pruritic papules spread over her trunk and extremities, and was diagnosed with LP. No mucosal lesions were observed. The diagnosis was histologically confirmed. Viral infection, contact with triggering substances, and use of medications were all ruled out. Treatment with sulfasalazine was begun and 5 months later, the lesions had disappeared. After a lesion-free period of 2 months, the patient was diagnosed with infiltrating ductal carcinoma in the left breast. She underwent a tumorectomy and was treated with chemotherapy and external radiotherapy of up to $60 \mathrm{~Gy}$ in the left breast, completing treatment after 2 months. Five months after terminating radiotherapy, the patient returned to our department, reporting a 4-month evolution of shiny violaceous papules clustered in the inframammary crease. The cutaneous lesions coincided exactly with the irradiated area (Fig. 1). A biopsy was performed and histopathological findings were consistent with LP. After 1 month of treatment with clobetasol propionate $0.05 \%$, the lesions disappeared.

The isomorphic Kóbner phenomenon appears in a variety of dermatoses, including LP. However, the appearance of LP confined to an irradiated site is extremely rare. Only two cases have been published by Kim and Krivda, ${ }^{1}$ and by Shurman et al. ${ }^{2}$ However, in our case, the radiotherapy induced the reappearance of the LP lesions in a patient who had previously presented with the disease and who had been free of lesions up to that moment. In our case, as in Kim and Krivda's, ${ }^{1}$ there was a 1-month latency period from the moment radiotherapy was completed to the appearance of LP.

T lymphocytes have been found to play a primordial role in the pathogenesis of LP. Furthermore, it is possible that the increase of adhesion molecules such as endothelial leukocyte adhesion molecule-1, intercellular adhesion molecule (ICAM)-1 and vascular cell adhesion molecule-1 via endothelial cells may also play an important role. ${ }^{3}$

The mechanism by which LP develops after radiotherapy is unknown. Radiotherapy is known to have immunomodulatory properties. Exposure to low doses of radiation modulates the function of both macrophages and $\mathrm{T}$ lymphocytes, and induces a selective enhancement of CD8+ T-cell responses. ${ }^{4}$ It has been shown that radiation increases the expression of proinflammatory molecules such as major histocompatibility complex (HMC), adhesion molecules (ICAM-1 and E-selectin in the endothelium) and cytokines, although the mechanism by which they are produced remains somewhat unclear. The 
immunomodulation process has been shown to last up to 6 months after completion of radiotherapy. The ICAM-1 levels probably rise markedly during treatment and gradually fall until they reach preradiotherapeutic levels 6 months after the end of treatment. This would not occur with E-selectin, the levels of which would increase during treatment, then decrease upon completion of radiotherapy, but would not return to baseline levels. ${ }^{5}$ Therefore, adhesion molecules may play an important role in the transendothelial migration of leucocytes and consequently in the inflammatory cell composition of the tissue. Radiation therefore most likely reproduces the inflammatory reaction involved in LP. Nevertheless, some studies have shown that low doses of fractionated radiotherapy may have anti-inflammatory effects and diminish the expression of adhesion molecules such as E-selectin. ${ }^{6}$ Interestingly, ionizing radiation was used in the past to treat certain inflammatory dermatoses.

We present a new case of LP induced by radiotherapy and propose that external radiotherapy may be another factor to consider as a cause of köbnerization in various dermatoses.

Conflict of interest: none declared.

\section{REFERENCES}

1. Kim JH, Krivda SJ. Lichen planus confined to a radiation therapy site. J Am Acad Dermatol 2002; 46: 604-5.

2. Shurman D, Reich HL, James WD. Lichen planus confined to a radiation field: the 'soradiotopic' response. J Am Acad Dermatol 2004; 50: 482-3.

3. Regezi JA, Dekker NP, MacPhail LA et al. Vascular adhesion molecules in oral lichen planus. Oral Surg Oral Med Oral Pathol Oral Radiol Endod 1996; 81: 682-90.

4. Pandey R, Shankar BS, Sharma D et al. Low dose radiation induced immunomodulation: effect on macrophages and CD8+ T cells. Int J Radiat Biol 2005; 81: 801-12.

5. Prott FJ, Handschel J, Micke $\mathrm{O}$ et al. Long-term alterations of oral mucosa in radiotherapy patients. Int J Radiat Oncol Biol Phys 2002; 54: 203-10.

6. Gaber MW, Sabek OM, Fukatsu K et al. Differences in ICAM1 and TNF- $\alpha$ expression between large single fraction and fractionated irradiation in mouse brain. Int J Radiat Biol 2003; 79: 359-66. 


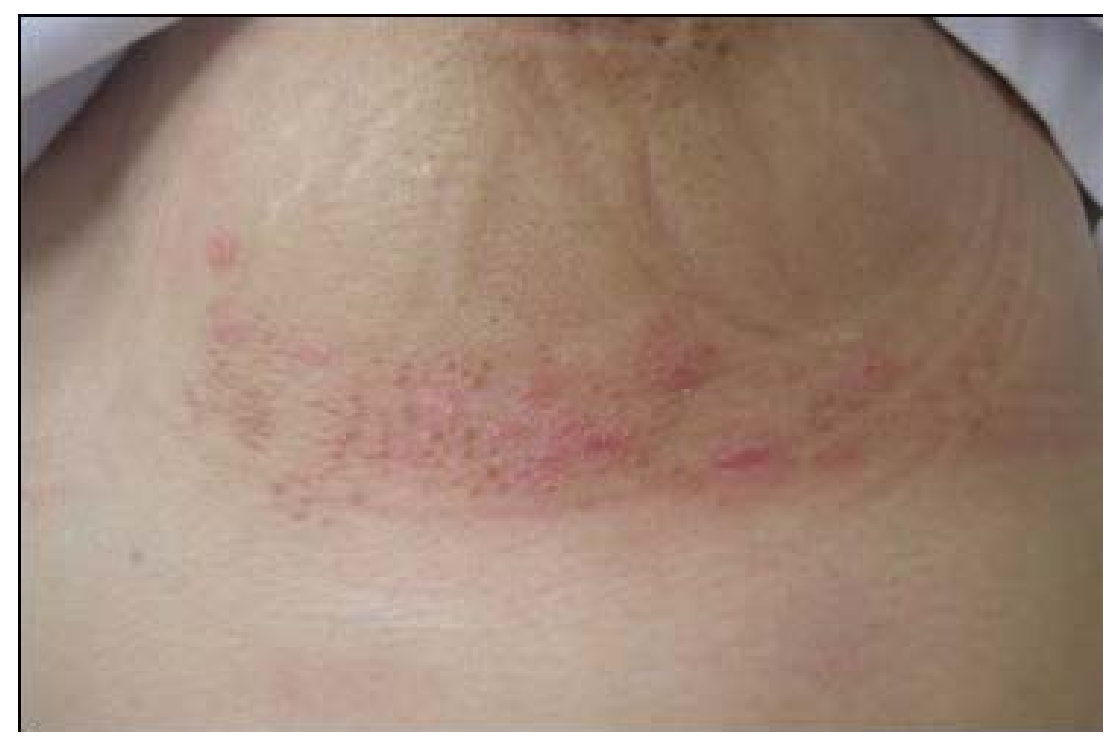

Figure 1. Violaceous polygonal papules clustered in the inframammary crease, the site treated by radiotherapy. 